\title{
Analysis of Equivalent Elastic Modulus of a Honeycomb Sandwich Considering Interference Effect with Face Sheet*
}

\author{
Dai-heng CHEN**, Takeshi MATSUMOTO** and Shingo OZAKI** \\ ** Department of Mechanical Engineering,Tokyo University of Science \\ Kagurazaka 1-3, Shinjuku-ku, Tokyo, 162-8601 Japan \\ E-mail: chend@rs.kagu.tus.ac.jp
}

\begin{abstract}
In this paper, in-plane equivalent elastic modulus $E_{y}$ of a hexagonal honeycomb sandwich, which includes the effect of face sheet interference, is studied by using numerical results of FEM. It is shown by comparing with deformation of practical honeycomb sandwich that there are two error factors to apply the rule of mixture to honeycomb sandwich. One of error factors is that the deformation of honeycomb core does not coincide with the face sheet since an inclined cell wall deforms much larger than a vertical cell wall. Another one is that the non-uniformity deformation of core along the height direction is induced by the interference effect with face sheet. Then, a method to calculate elastic modulus based on the compatibility condition of core and face sheet is proposed, and its validity is verified by using numerical results of FEM.
\end{abstract}

Key words : Honeycomb Sandwich, Equivalent Elastic Modulus, Height Effect, Compatibility Condition, FEM

\section{Introduction}

Nowadays, a honeycomb sandwich attracts attention as a composite material which can meet a high performance need in mechanical design. When evaluating the strength of honeycomb sandwich, which is used in a practical analysis as a virtually flat plate, the equivalent elastic modulus of periodic cell structures are necessary for design. There have been many theoretical studies about the equivalent elastic modulus of cores which constitutes the honeycomb sandwich, and many theoretical formulas have been presented ${ }^{(1) \sim(4)}$. However, there are few studies about the equivalent elastic modulus, in which the influence of height of honeycomb sandwich is taken into account. Becker ${ }^{(5),(6)}$, Hohe and Becker ${ }^{(7)}$, Chen and Davalos ${ }^{(8)}$ presented methods for analyzing the in-plane equivalent elastic modulus of core only. In their studies, the face sheet is virtually treated as a condition of displacement-constraint, and the deformation of core is fit to the face sheet, so that the influence of face sheet thickness and the interaction between the face sheet and core are neglected. In this paper, we study a theoretical

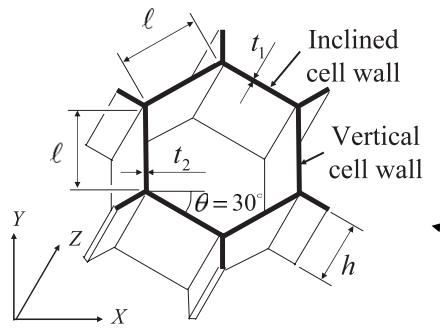

(a)

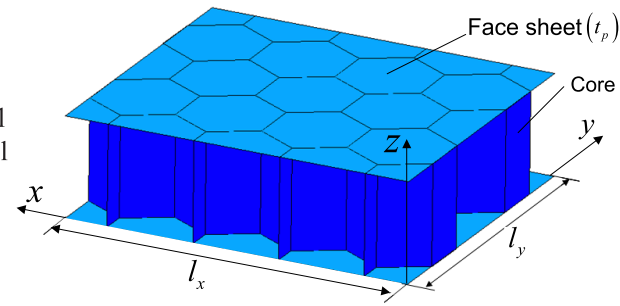

(b)

Fig. 1 Geometry of honeycomb sandwich: (a) regular hexagonal honeycomb core; (b) analyzed model of honeycomb sandwich 
analysis of the in-plane equivalent elastic modulus of a honeycomb sandwich considering the interference effect between the core and face sheet, as shown in Fig.1(b). Model of Fig.1(b) consists of face sheets above and below and a core part, which consists of periodically arrayed regular hexagonal cells shown in Fig.1(a). First, we calculate the equivalent elastic modulus of the honeycomb sandwich based on the law of mixture ${ }^{(9)}$ about a composite material, and show an accuracy problem of the law of mixture for the honeycomb sandwich by comparing its results with numerical results of FEM. Next, we present a new method of theoretical analysis of the in-plane equivalent elastic modulus considering the compatibility condition of the core and face sheet, and show the validity by using numerical results of FEM.

Analyzed models in Fig.1(a) and Fig.1(b) are characterized by the core height $h$, the cell wall length $l$, the cell wall thickness $t_{1}$ (inclined cell wall), $t_{2}$ (vertical cell wall) and the face sheet thickness $t_{p}$. And, the core and face sheet are assumed as isotropic homogeneous material with Young's modulus and Poisson ratio noted by $E_{s}, v_{s}$ and $E_{p}, v_{p}$ respectively.

\section{Analysis method of finite element method}

In this study, we use numerical results of FEM by commercial software MSC.Marc ${ }^{(10)}$ about the equivalent elastic modulus of the honeycomb sandwich to show the validity of the presented method. In the analysis of FEM, the analyzed model of honeycomb sandwich in Fig.1(b) has length of $x$-direction $l_{x}=(2 l \cos \theta) \times 4$, and length of $y$-direction $l_{y}=l(1+\sin \theta) \times$ 4. Every cell walls and face sheets are discretized using 100 and 25000, respectively, bilinear thick shell elements. In boundary conditions, every nodes of $y=0$ (refer to coordinates in Fig.1(b)) are fixed at $y$-direction, every nodes of $y=l_{y}$ are given an imposed displacement $u_{y}=\Delta U_{y}$ in $y$-direction, every nodes of $y=0$ and $x=3 l \cos \theta$ are fixed in $x$-direction, every nodes of $z=h / 2$ are fixed in $z$-direction. In the numerical analysis, as far as there isn't a special specification, material properties of the face sheet and cell wall are taken as $E_{s}=E_{p}=E, v_{s}=v_{p}=0.3$, and geometric properties are taken as $t_{1}=t_{2}=t, \theta=30^{\circ}$.

As an example, Fig.2 shows numerical results of distribution of reaction forces $F_{i}$ on the node $i$ of face sheet for sandwich with $l=10 \mathrm{~mm}, t / l=0.05, h / l=1.0, t_{p} / t=0.5$, $E=200 \mathrm{GPa}$ in $\Delta U_{y}=l_{y} \times 0.01$. It is seen from Fig.2 that the reaction forces $F_{i}$ at $x=0$ and $x=l_{x}$ are higher than those in central region by about $10 \%$ because of the influence of boundary in both sides. However, the influence is local. For example, the ratio of $F_{A B}, F_{B C}$ and $F_{C D}$, which are summations of the reaction force $F_{i}$ from $\mathrm{A}$ to $\mathrm{B}$, from $\mathrm{B}$ to $\mathrm{C}$ and from $\mathrm{C}$ to D respectively, is $F_{A B}: F_{B C}: F_{C D}=0.99: 1: 0.99$. So, it seems that the reaction force of each node from $\mathrm{B}$ to $\mathrm{C}$ is hardly influenced by the boundary. Also numerical results of FEM show that the summation of reaction force $F_{B C}$ is almost constant in changing the number of units in $y$-direction. For these reasons, in our study, summation of the reaction force $F_{i}$ of core and face sheet from B to $\mathrm{C}$ at the center, denoted by $R_{B C}$, is used to calculate the $y$-direction Young's modulus of honeycomb sandwich as follows.

$$
E_{y, F E M}=\frac{R_{B C}}{\left[2 l \cos \theta\left(2 t_{p}+h\right)\right] \times \frac{\Delta U_{y}}{l_{y}}}
$$

Also, the equivalent elastic modulus is determined using stress due to a unit strain in a unit volume, and, as will be shown later, the results are almost the same. Here after, the equivalent elastic modulus of honeycomb sandwich obtained from Eq.(1) is written as $E_{y, F E M}$.

Moreover, the validity of $E_{y, F E M}$ by Eq.(1) can be shown by comparing it with the known solution for core only without face sheet. For example, Eq.(1) gives a value of $E_{y, F E M}$ for the model used in Fig. 2 with the face sheet thickness $t_{p}=10^{-10} \times t$ corresponding to the known theoretical solution ${ }^{(3),(4)}$ well by not more than $0.5 \%$. 


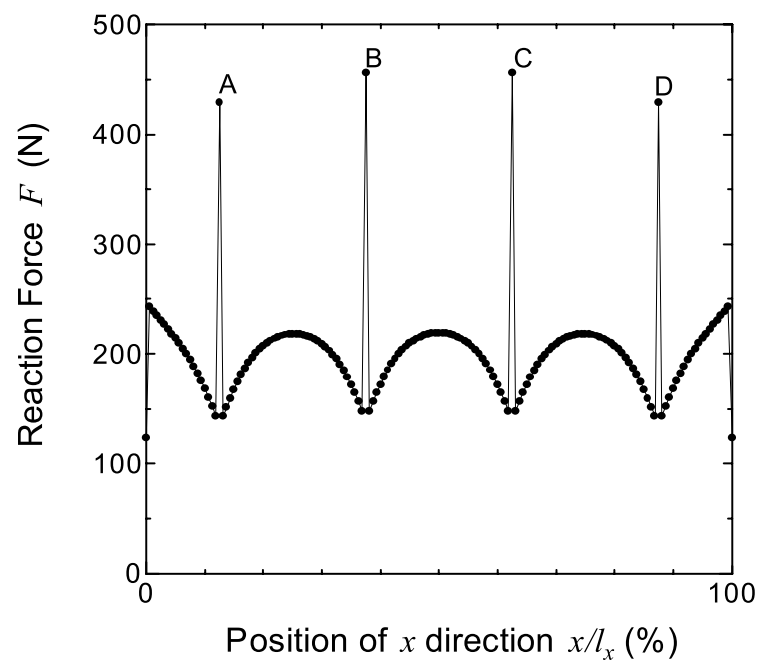

Fig. 2 Distribution of reaction forces on the $y=l_{y}$ nodes of face sheet

\section{Investigation of equivalent elastic modulus $E_{y}$}

\subsection{Equivalent elastic modulus given by the law of mixture}

By considering the honeycomb sandwich as a composite material made of the face sheet and core, the equivalent elastic modulus $E_{y}$ is obtained as following equation by the law of mixture $^{(9)}$

$$
E_{y, \text { com }}=\frac{2 E_{p} t_{p}+E_{\text {core }} h}{2 t_{p}+h}
$$

where $E_{\text {core }}$ is the $y$-direction equivalent elastic modulus of core only, and it is obtained as follows ${ }^{(3)}$ :

$$
E_{\text {core }}=\frac{(1+\sin \theta)}{\cos ^{3} \theta}\left(\frac{t_{1}}{l}\right)^{3} E_{s} \frac{1}{\left[1+\left\{2\left(1+v_{s}\right)+\tan ^{2} \theta+\frac{2 t_{1}}{t_{2} \cos ^{2} \theta}\right\}\left(\frac{t_{1}}{l}\right)^{2}\right]} .
$$

Here after, the equivalent elastic modulus $E_{y}$ given by Eq.(2) is written as $E_{y, c o m}$.

Figure 3 and Fig.4 show the ratio of the equivalent elastic modulus $E_{y, c o m}$ to numerical results $E_{y, F E M}$ obtained from FEM by dash line for the honeycomb sandwich with $t / l=0.05$. In Fig.3, the face sheet thickness $t_{p}$ is changed and $h / l$ is set as $h / l=0.25,1$ and 2 . And in Fig.4, the core height $h$ is changed for three kinds of $t_{p} / t=0.5,1$ and 2. It is seen from these figures that in the considered dimension range, the thinner the face sheet thickness is and the higher the core height is, the bigger the error of equivalent elastic modulus of the law of mixture, compared to the numerical result of FEM, grows, and the utmost error may be about $20 \%$. Here the range of face sheet thickness is taken to be $t_{p} / t=0.5 \sim 2$ by considering that the honeycomb sandwich is practically used as $t_{p} \geq t$. Actually, however, we investigated more wide range of $t_{p} / t$ as $t_{p} / t=0 \sim 2$ including the thickness of face sheet becomes 0 . The error of $E_{y, c o m}$ compared to $E_{y, F E M}$ becomes maximum (about $40 \%$ ) at about $t_{p} / t=0.01$, and it becomes smaller as the face sheet thickness becomes so that the value of $t_{p} / t$ leaves from 0.01 . In such honeycomb sandwiches, the ratio of core rigidity $E_{\text {core }} h$ to the face sheet rigidity $2 E_{p} t_{p}$ is about 3 at $t_{p} / t=0.01$, but the ratio is over 160 at $t_{p} / t=0.5 \sim 2$. Based on this fact, it seems that the analytical error of the law of mixture becomes the biggest when the order of core rigidity $E_{c o r e} h$ is almost equal to that of face sheet $2 E_{p} t_{p}$. So, the smaller the thickness of face sheet $t_{p}$ is, the bigger the error becomes in the range of Fig.3. 


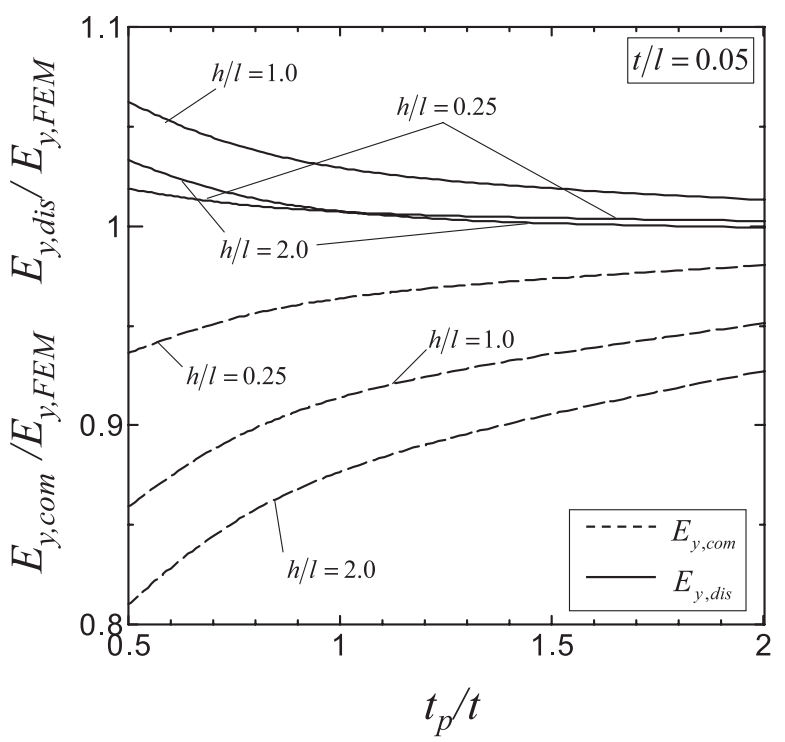

Fig. 3 Relation of $E_{y, c o m} / E_{y, F E M}$ versus $t_{p} / t$ for honeycomb with $h / l=1, t / l=0.05$

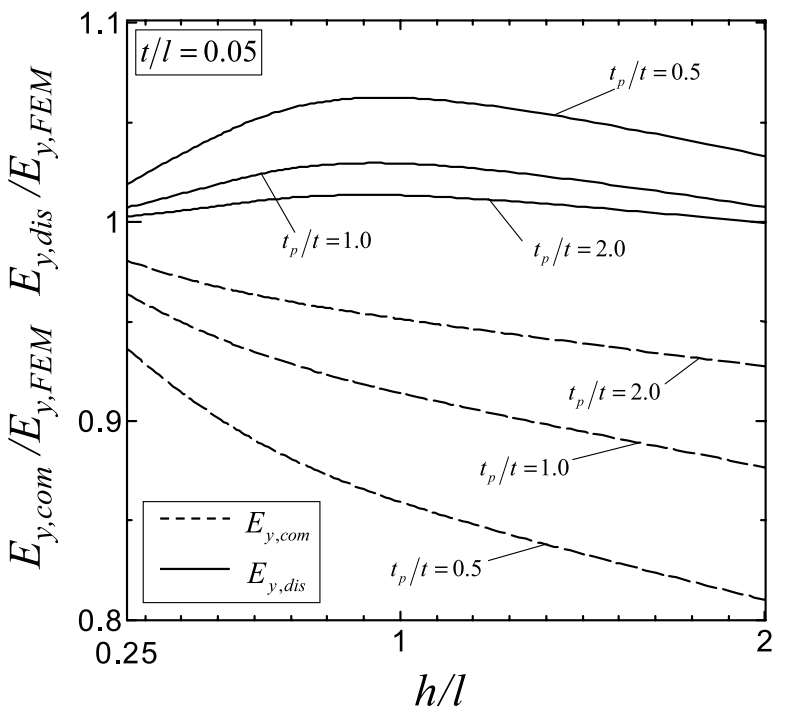

Fig. 4 Relation of $E_{y, \text { com }} / E_{y, F E M}$ versus $h / l$ for honeycomb with $t_{p} / t=0.5, t / l=0.05$

The reason of analytic error due to the law of mixture is that there is a difference of rigidity about $y$-direction deformation between the vertical cell wall and inclined cell wall which constitute the honeycomb. Namely as shown in Fig. 5 which shows 1 unit (one vertical cell wall, two inclined cell walls and two face sheets above and below these cell walls), the core is made of the vertical cell wall and inclined cell wall, and the equivalent elastic modulus of vertical cell wall (noted by $E_{y, v}$ ) and inclined cell wall (noted by $E_{y, i}$ ) are expressed as follows ${ }^{(3)}$.

$$
\begin{aligned}
& E_{y, v}=E_{s} \frac{t_{2}}{2 l \cos \theta} \\
& E_{y, i}=E_{s} \frac{\sin \theta}{\cos ^{3} \theta}\left(\frac{t_{1}}{l}\right)^{3} \frac{1}{\left[1+\left\{2\left(1+v_{s}\right)+\tan ^{2} \theta\right\}\left(\frac{t_{1}}{l}\right)^{2}\right]}
\end{aligned}
$$

The equivalent elastic modulus of inclined cell wall $E_{y, i}$ is lower than that of vertical cell wall $E_{y, v}$, because the bending deformation occurs in the inclined cell wall only (for example the 
ratio between both of the inclined and vertical cell walls is about $E_{y, i}: E_{y, v}=1: 77$ for a case of $t_{p} / t=1.0, h / l=1.0$ in Fig.4). As a result, in the case of core only, the ratio of the deformation of inclined cell wall to the total deformation is very high, but in the case of honeycomb sandwich, its deformation is restrained by the interference with face sheet. Figure6 shows the deformed configuration around connection between the inclined cell wall and vertical cell wall when the honeycomb sandwich with $t_{p} / t=0.5, h / l=1.0$ is deformed homogeneously in $y$-direction. It is seen from Fig. 6 that the deformation of core changes along with the distance from the face sheet, because of the interference with the face sheet. For this reason, the equivalent elastic modulus of core, obtained as two-dimensional deformation like Eq.(3), can't apply rigorously to problems of such core where three-dimensional deformation occurs as shown in Fig.6.

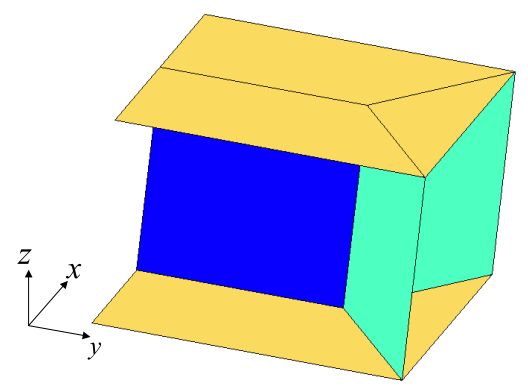

Fig. 5 Representative unit of honeycomb sandwich

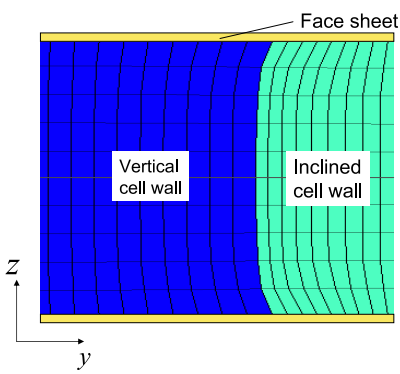

Fig. 6 Deformed cross-section of honeycomb with $t_{p} / t=0.5, h / l=1$ and $t / l=0.05$ subjected to in-plane loading

\subsection{Equation based on the compatibility condition}

From the investigation of the previous section, it is understood that the law of mixture can't apply to evaluating the equivalent elastic modulus of the honeycomb sandwich consisting of cores and face sheets, due to the low accuracy of analysis, because of the large difference between rigidities of inclined cell wall and vertical cell wall of core. So, we present a new method of theoretical analysis for evaluating equivalent elastic modulus of composite shown in Fig.7 which is made of 3 materials of different elastic property (core is material 1, 2 and face sheets are material 3, and each material's Young's modulus is denoted by $E_{1}, E_{2}$ and $E_{3}$ respectively). $h, b, h_{3}, l_{1}$ and $l_{2}$ in Fig.7 are initial dimensions of model before deformation.

To consider the interference effect between the core and face sheet, we divide the deformation of sandwich model of Fig.7, caused by a displacement $\delta$ homogeneously applied at one edge, into two phases shown in Fig.8(a) and (b) respectively. At phase 1, by assuming that there is no combination between the core and face sheet, both core and face sheet can deform freely respectively. In this phase, the point $\mathrm{M}$, which is intersection of the face sheet and the boundary of material 1 and 2 , will go to a point $\mathbf{M}_{c}$ at the core and go to a point $\mathbf{M}_{p}$ at the face sheet after deforming. $\delta_{c}$ and $\delta_{p}$ in Fig.8(a) are distance of each point, and they are 
different from each other as followings.

$$
\delta_{c}=\delta \frac{l_{2} / E_{2}}{l_{2} / E_{2}+l_{1} / E_{1}}, \quad \delta_{p}=\delta \frac{l_{2}}{l_{2}+l_{1}}
$$

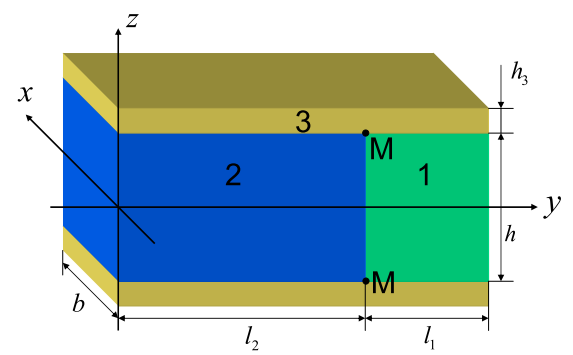

Fig. 7 Sandwich model consisted by 3 kinds of homogeneous materials

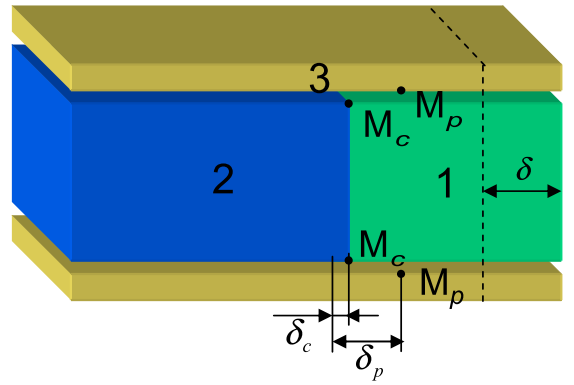

(a)

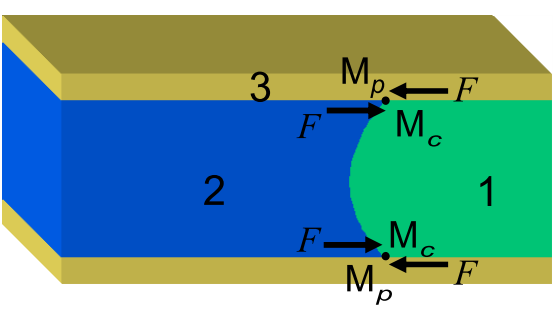

(b)

Fig. 8 Deformation process of sandwich model: (a) Deformation of each materials under non-constrained condition; (b) Deformation under interference effect

Figure 8(a) shows that $\delta_{c}$ is smaller than $\delta_{p}$ if $E_{1}<E_{2}$. That is to say, because material 1 deforms larger than material 2 for small Young's modulus, inconsistency of deformation occurs between the face sheet which deform homogeneously and the core which consist of material 1 and 2, then the point $M$ which is the unique point of junction divides into $\mathbf{M}_{c}$ and $\mathrm{M}_{p}$ after deformation. However the points $\mathrm{M}_{c}$ and $\mathrm{M}_{p}$ have to be the same point by restraint of junction, and actual deformation of the core in $y$-direction becomes inhomogeneous along the height as shown in Fig.6. So, in phase 2 shown in Fig.8(b), in order to meet compatibility condition between the core and face sheet, two forces $F$, simulating the constraint between the face sheet and core, are respectively applied to the core's point $\mathbf{M}_{c}$ and the face sheet's point $\mathrm{M}_{p}$, obtained in phase1 under an assumption of both face sheet and core freely deformating, so that the points $\mathrm{M}_{p}$ and $\mathrm{M}_{c}$ move together to an identical point. Here the forces $F$ are distributed homogeneously along $x$-direction of width, and $F$ is defined as a density per unit width. The force $F$ is determined to meet the following equation, where $u_{y p}$ and $u_{y c}$ are deformation values of the point $\mathrm{M}_{p}$ of face sheet and the point $\mathrm{M}_{c}$ of core respectively due to the force $F$.

$$
\delta_{c}+u_{y c}=\delta_{p}-u_{y p}
$$

The face sheet is so thin that the deformation $u_{y p}$ of point $\mathrm{M}_{p}$ due to the force $F$ can be obtained from a deformation problem of one-dimension's bar fixed at both ends shown in Fig.9. As a result, $u_{y p}$ is

$$
u_{y p}=\frac{F l_{1} l_{2}}{E_{3} h_{3}\left(l_{1}+l_{2}\right)} .
$$




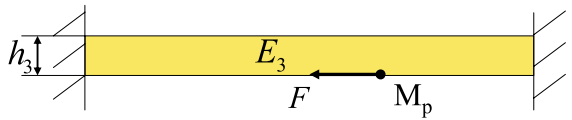

Fig. 9 Force acting on material 3

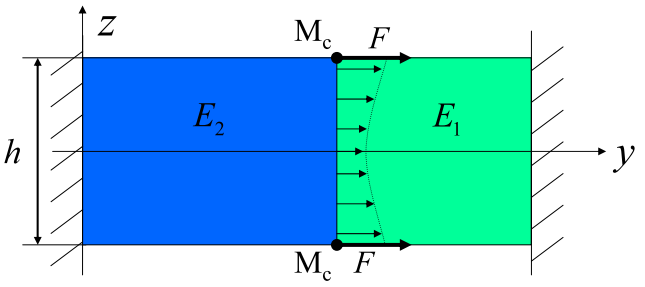

Fig. 10 Forces acting on materials 1 and 2

In order to obtain the deformation $u_{y c}$ of point $\mathrm{M}_{c}$ due to the force $F$, consider a problem of the force $F$ acting at the point $\mathrm{M}_{c}$ in the composite material of material 1 and 2 under condition that both ends are fixed in Fig.10. As an approximation analysis, suppose that the deformation field in material 1 and 2 due to the force $F$ can be expressed as follows:

$$
\begin{cases}u_{y 1}=\left[1-\frac{y-l_{2}}{l_{1}}\right] A_{2} l_{2} \cosh \left(\frac{2 \lambda}{h} z\right) & \text { for region } 1 \\ u_{y 2}=A_{2} y \cosh \left(\frac{2 \lambda}{h} z\right) & \text { for region 2 }\end{cases}
$$

where $A_{2}$ and $\lambda$ are unknown. These unknowns are obtained by minimizing the total potential energy of system $\Pi$, namely from the following equations:

$$
\frac{\partial \Pi}{\partial A_{2}}=0, \quad \frac{\partial \Pi}{\partial \lambda}=0 .
$$

The total potential energy of system $\Pi$ is given by

$$
\Pi=U_{1}+U_{2}-2 F b A_{2} l_{2} \cosh (\lambda),
$$

where $U_{1}$ and $U_{2}$ are strain energy of material 1 and 2 respectively, and they are calculated from the supposed deformation field Eq.(9) as follows

$$
\begin{aligned}
U_{1}= & \frac{E_{1} b l_{2}^{2} A_{2}^{2}}{2 l_{1}^{2}}\left(\frac{h}{2 \lambda}\right)[\cosh (\lambda) \sinh (\lambda)+\lambda] l_{1} \\
& +\frac{G_{1} b l_{2}^{2} A_{2}^{2}}{2 l_{1}^{2}}\left(\frac{2 \lambda}{h}\right)[\cosh (\lambda) \sinh (\lambda)-\lambda] \frac{l_{1}^{3}}{3} \\
U_{2}= & \frac{E_{2} b A_{2}^{2}}{2}\left(\frac{h}{2 \lambda}\right)[\cosh (\lambda) \sinh (\lambda)+\lambda] l_{2} \\
& +\frac{G_{2} b A_{2}^{2}}{2}\left(\frac{2 \lambda}{h}\right)[\cosh (\lambda) \sinh (\lambda)-\lambda] \frac{l_{2}^{3}}{3},
\end{aligned}
$$

where $G_{1}$ and $G_{2}$ are shear modulus of material 1 and 2 respectively. Substituting Eq.(12) and Eq.(13) into Eq.(11), an equation for deciding the unknown $\lambda$ is obtained from Eq.(10) as follows.

$$
\begin{aligned}
f_{1}(\lambda) \sinh (\lambda)-f_{2}(\lambda) \cosh (\lambda)=0 \\
f_{1}(\lambda)=\left\{\frac{E_{1} l_{1}}{2}\left(\frac{l_{2}}{l_{1}}\right)^{2}+\frac{E_{2} l_{2}}{2}\right\}\left(\frac{h}{2 \lambda}\right) g_{1}(\lambda) \\
+\left\{\frac{G_{1} l_{1}^{3}}{6}\left(\frac{l_{2}}{l_{1}}\right)^{2}+\frac{G_{2} l_{2}^{3}}{6}\right\}\left[\left(\frac{2 \lambda}{h}\right) g_{2}(\lambda)\right]
\end{aligned}
$$




$$
\begin{aligned}
f_{2}(\lambda)= & \left\{\frac{E_{1} l_{1}}{4}\left(\frac{l_{2}}{l_{1}}\right)^{2}+\frac{E_{2} l_{2}}{4}\right\}\left[-\frac{h}{2 \lambda^{2}} g_{1}(\lambda)+\left(\frac{h}{2 \lambda}\right)\left\{2 \cosh ^{2}(\lambda)\right\}\right] \\
& +\left\{\frac{G_{1} l_{1}^{3}}{12}\left(\frac{l_{2}}{l_{1}}\right)^{2}+\frac{G_{2} l_{2}^{3}}{12}\right\}\left[\frac{2}{h} g_{2}(\lambda)+\frac{2 \lambda}{h}\left\{2 \sinh ^{2}(\lambda)\right\}\right] .
\end{aligned}
$$

And

$$
\left\{\begin{array}{l}
g_{1}(\lambda)=\cosh (\lambda) \sinh (\lambda)+\lambda \\
g_{2}(\lambda)=\cosh (\lambda) \sinh (\lambda)-\lambda
\end{array} .\right.
$$

By deciding $\lambda$ from Eq.(14), $A_{2}$ is given by

$$
A_{2}=\frac{F l_{2} \cosh (\lambda)}{f_{1}(\lambda)}
$$

So, from Eq.(9) the deformation $u_{y c}$ of point $\mathrm{M}_{c}$ due to force $F$ is

$$
u_{y c}=A_{2} l_{2} \cosh (\lambda)=F \frac{l_{2}^{2} \cosh ^{2}(\lambda)}{f_{1}(\lambda)} .
$$

And the force $F$ is decided by substituting Eq.(19) and Eq.(8) into Eq.(7).

$$
F=\frac{\delta_{p}-\delta_{c}}{\frac{l_{2}^{2} \cosh ^{2}(\lambda)}{f_{1}(\lambda)}+\frac{l_{1} l_{2}}{E_{3} h_{3}\left(l_{1}+l_{2}\right)}}
$$

From the description of above, unknowns $F, A_{2}$ and $\lambda$ can be determined. And, the final deformation field in each material is obtained by adding the deformation field before the action of the force $F$ to the deformation field due to the force $F$. They are shown as follows. The deformation field in material 1 is

$$
u_{y 1}=\delta_{c}+\frac{\delta-\delta_{c}}{l_{1}}\left(y-l_{2}\right)+A_{2} l_{2}\left[1-\frac{y-l_{2}}{l_{1}}\right] \cosh \left(\frac{2 \lambda}{h} z\right)
$$

the deformation field in material 2 is

$$
u_{y 2}=\frac{\delta_{c}}{l_{2}} y+A_{2} y \cosh \left(\frac{2 \lambda}{h} z\right)
$$

and the deformation field in material 3 is

$$
\left\{\begin{array}{ll}
u_{y 3}=\frac{\delta_{p}+u_{y p}}{l_{2}} y & \text { for } 0 \leq y \leq l_{2} \\
u_{y 3}=\left(\delta_{p}+u_{y p}\right)+\frac{\delta-\left(\delta_{p}+u_{y p}\right)}{l_{1}}\left(y-l_{2}\right) & \text { for } l_{2} \leq y \leq l_{1}+l_{2}
\end{array} .\right.
$$

Also the corresponding strain energy of total system $\bar{U}$ can be obtained from these deformation fields. So, the equivalent elastic modulus $E_{y}$ of the sandwich structure shown in Fig.7 is given by

$$
E_{y}=\frac{2 \bar{U}}{V\left(\frac{\delta}{l_{1}+l_{2}}\right)^{2}},
$$

where $V$ is the unit volume of sandwich material and is calculated by using following equation:

$$
V=b\left(l_{1}+l_{2}\right)\left(2 h_{3}+h\right) .
$$

Here after, the equivalent elastic modulus $E_{y}$ given by Eq.(24) is written as $E_{y, d i s}$.

In order to confirm the validity of this theoretical analysis at first, the equivalent elastic modulus for the sandwich model shown in Fig.7, in which $h_{3} / h=0.05, l_{1} / l_{2}=0.5$ and $E_{3}$ is fixed, $E_{1}, E_{2}$ are varied, were analyzed by FEM, Eq.(2) and Eq.(24), whose results are denoted by $E_{y, F E M}, E_{y, c o m}$ and $E_{y, d i s}$ respectively. Table1 shows comparisons for various ratio of $E_{1}$ to $E_{3}$ and $E_{3}$ to $E_{2}$. In addition, numeric values in [ ] are the relative error of $E_{y, \text { com }}$ and $E_{y, d i s}$ to $E_{y, F E M}$. It is seen from the table that analysis accuracy changes with condition of material, but the error of the analysis method presented in our study is smaller than that of the law of mixture in any cases. 
Table 1 Young's modulus $E_{y}$ for sandwich model of 3 kinds of homogeneous materials

\begin{tabular}{|c|c|c|c|c|}
\hline$E_{1} / E_{2}$ & $E_{3} / E_{2}$ & $E_{y, F E M} / E_{3}$ & $E_{y, \text { com }} / E_{3}$ & $E_{y, \text { dis }} / E_{3}$ \\
\hline \multirow{6}{*}{0.5} & \multirow{2}{*}{2} & \multirow{2}{*}{$4.48 \times 10^{-1}$} & $4.32 \times 10^{-1}$ & $4.39 \times 10^{-1}$ \\
\hline & & & {$[-3.72 \%]$} & {$[-2.09 \%]$} \\
\hline & \multirow{2}{*}{10} & \multirow{2}{*}{$1.64 \times 10^{-1}$} & $1.59 \times 10^{-1}$ & $1.62 \times 10^{-1}$ \\
\hline & & & {$[-2.70 \%]$} & {$[-0.87 \%]$} \\
\hline & \multirow{2}{*}{100} & \multirow{2}{*}{$9.83 \times 10^{-2}$} & $9.77 \times 10^{-2}$ & $9.81 \times 10^{-2}$ \\
\hline & & & {$[-0.53 \%]$} & {$[-0.14 \%]$} \\
\hline \multirow{6}{*}{0.1} & \multirow{2}{*}{2} & \multirow{2}{*}{$2.60 \times 10^{-1}$} & $2.05 \times 10^{-1}$ & $2.61 \times 10^{-1}$ \\
\hline & & & {$[-21.5 \%]$} & {$[0.05 \%]$} \\
\hline & \multirow{2}{*}{10} & \multirow{2}{*}{$1.32 \times 10^{-1}$} & $1.14 \times 10^{-1}$ & $1.34 \times 10^{-1}$ \\
\hline & & & [13.9\%] & {$[1.20 \%]$} \\
\hline & \multirow{2}{*}{100} & \multirow{2}{*}{$9.55 \times 10^{-2}$} & $9.32 \times 10^{-2}$ & $9.56 \times 10^{-2}$ \\
\hline & & & {$[-2.38 \%]$} & {$[0.15 \%]$} \\
\hline \multirow{6}{*}{0.01} & \multirow{2}{*}{2} & \multirow{2}{*}{$1.82 \times 10^{-1}$} & $1.04 \times 10^{-1}$ & $1.93 \times 10^{-1}$ \\
\hline & & & [-42.6\%] & {$[6.24 \%]$} \\
\hline & \multirow{2}{*}{10} & \multirow{2}{*}{$1.20 \times 10^{-1}$} & $9.36 \times 10^{-2}$ & $1.24 \times 10^{-1}$ \\
\hline & & & {$[-21.8 \%]$} & [3.49\%] \\
\hline & \multirow{2}{*}{100} & \multirow{2}{*}{$9.44 \times 10^{-2}$} & $9.12 \times 10^{-2}$ & $9.48 \times 10^{-2}$ \\
\hline & & & {$[-3.40 \%]$} & {$[0.41 \%]$} \\
\hline
\end{tabular}

\subsection{Analysis of elastic modulus of haneycomb sandwich based on compatibility condi-} tion

In order to apply the new analysis method presented in the foregoing section to the analysis of equivalent elastic modulus of the honeycomb sandwich, the properties of material 3 are set as $E_{3}=E_{p}$ and $h_{3}=t_{p}$ relating to the face sheet. In a similar way, material 1 and 2 are corresponding to the inclined cell wall and vertical cell wall respectively as following equation,

$$
\left\{\begin{array}{lll}
E_{1}=E_{y, i} & \text { of } & \text { Eq. }(4) \\
E_{2}=E_{y, v} & \text { of } & \text { Eq.(5) }
\end{array}\right.
$$

and geometric properties are $l_{1}=l \sin \theta, l_{2}=l$, and $b=2 l \cos \theta$. In addition, the antiplane modulus of rigidity $G_{y z, i}$ of inclined cell wall and the antiplane modulus of rigidity $G_{y z, v}$ of vertical cell wall, which are equal to the modulus of rigidity $G_{1}$ and $G_{2}$ of material 1 and 2, are obtained as follows by the study of Gibson and Ashby ${ }^{(3)}$.

$$
\left\{\begin{array}{l}
G_{1}=G_{y z, i}=G_{s} \frac{\sin \theta}{\cos \theta}\left(\frac{t_{1}}{l}\right) \\
G_{2}=G_{y z, v}=G_{s} \frac{t_{2}}{2 l \cos \theta}
\end{array}\right.
$$

In this way, the elastic modulus $E_{y, d i s}$ of the honeycomb sandwich is calculated by replacing the materials of the composite shown in Fig.7 by the inclined cell wall, vertical cell wall and face sheet respectively.

Figure 3 and Fig. 4 also show the ratio of result $E_{y, d i s}$ of the theoretical analysis presented in our study to numerical results by FEM $E_{y, F E M}$ (solid line in fig). In addition, table $2 \sim 4$ show $E_{y, F E M}, E_{y, c o m}$ and $E_{y, d i s}$, obtained from FEM, Eq.(2) and Eq.(24) respectively, for various systematically changed geometric properties and material property systematically. Common geometric properties used in 3 tables are: the cell wall thickness $t / l=0.01,0.05,0.10$ and the cell wall height $h / l=0.5,1.0,2.0$. Table 2 shows the case of regular hexagonal honeycomb sandwich of $t_{p}=t_{1}=t_{2}=t$. Table 3 shows the case of $t_{p}=t_{1}=t, t_{1} / t_{2}=1 / 2$, and table 4 shows the case that Young's modulus of the face sheet $E_{p}$ is twice as much as that of core material $\left(E_{p} / E_{s}=2\right)$. In these tables relative errors of Eq.(24) and Eq.(2) compared to $E_{y, F E M}$ are shown by numeric value in [ ] . 
It is seen from these figures and tables that analysis accuracy of the equivalent elastic modulus $E_{y, d i s}$ of honeycomb sandwich presented in our study is superior to that of the law of mixture, and its error is less than $5 \%$ except for the case of very thin face $\operatorname{sheet}\left(t_{p} / t=0.5\right.$ ). The main reason of low accuracy for thin face sheet seems to be caused by antiplane warpage occurring at the thin face sheet by the interference with core.

Table 2 Young's modulus $E_{y} / E_{s}$ for regular hexagonal honeycomb with $t_{p}=t_{1}=t_{2}=$ $t$

\begin{tabular}{|c|c|c|c|c|}
\hline$t / l$ & $h / l$ & $E_{y, F E M} / E_{s}$ & $E_{y, \text { com }} / E_{s}$ & $E_{y, d i s} / E_{s}$ \\
\hline \multirow{5}{*}{0.01} & 0.50 & $4.08 \times 10^{-2}$ & $\begin{array}{c}3.85 \times 10^{-2} \\
{[-5.67 \%]}\end{array}$ & $\begin{array}{c}4.17 \times 10^{-2} \\
{[2.19 \%]}\end{array}$ \\
\cline { 2 - 5 } & 1.00 & $2.14 \times 10^{-2}$ & $\begin{array}{c}1.96 \times 10^{-2} \\
{[-8.49 \%]}\end{array}$ & $\begin{array}{c}2.21 \times 10^{-2} \\
{[3.28 \%]}\end{array}$ \\
\cline { 2 - 5 } & 2.00 & $1.13 \times 10^{-2}$ & $\begin{array}{c}9.90 \times 10^{-3} \\
{[-12.34 \%]}\end{array}$ & $\begin{array}{c}1.14 \times 10^{-2} \\
{[1.03 \%]}\end{array}$ \\
\hline \multirow{5}{*}{0.05} & 1.00 & $9.98 \times 10^{-2}$ & $\begin{array}{c}9.12 \times 10^{-2} \\
{[-8.59 \%]}\end{array}$ & $\begin{array}{c}1.03 \times 10^{-1} \\
{[2.94 \%]}\end{array}$ \\
\cline { 2 - 5 } & 0.50 & $1.77 \times 10^{-1}$ & $\begin{array}{c}1.67 \times 10^{-1} \\
{[-5.83 \%]}\end{array}$ & $\begin{array}{c}1.81 \times 10^{-1} \\
{[1.89 \%]}\end{array}$ \\
\cline { 2 - 5 } & 2.00 & $5.46 \times 10^{-2}$ & $\begin{array}{c}4.79 \times 10^{-2} \\
{[-12.31 \%]}\end{array}$ & $\begin{array}{c}5.51 \times 10^{-2} \\
{[0.75 \%]}\end{array}$ \\
\hline \multirow{5}{*}{0.1} & 0.50 & $3.05 \times 10^{-1}$ & $\begin{array}{c}2.87 \times 10^{-1} \\
{[-5.85 \%]}\end{array}$ & $\begin{array}{c}3.10 \times 10^{-1} \\
{[1.51 \%]}\end{array}$ \\
\cline { 2 - 5 } & 1.00 & $1.84 \times 10^{-1}$ & $\begin{array}{c}1.69 \times 10^{-1} \\
{[-8.46 \%]}\end{array}$ & $\begin{array}{c}1.89 \times 10^{-1} \\
{[2.45 \%]}\end{array}$ \\
\cline { 2 - 5 } & 2.00 & $1.06 \times 10^{-1}$ & $\begin{array}{c}9.31 \times 10^{-2} \\
{[-11.83 \%]}\end{array}$ & $\begin{array}{c}1.06 \times 10^{-1} \\
{[0.43 \%]}\end{array}$ \\
\hline
\end{tabular}

Table 3 Young's modulus $E_{y} / E_{s}$ for regular hexagonal honeycomb with $t_{p}=t_{1}=$ $t, t_{2} / t_{1}=2$

\begin{tabular}{|c|c|c|c|c|}
\hline$t / l$ & $h / l$ & $E_{y, F E M} / E_{s}$ & $E_{y, \text { com }} / E_{s}$ & $E_{y, d i s} / E_{s}$ \\
\hline \multirow{3}{*}{0.01} & 0.50 & $4.05 \times 10^{-2}$ & $\begin{array}{c}3.85 \times 10^{-2} \\
{[-5.01 \%]}\end{array}$ & $\begin{array}{c}4.16 \times 10^{-2} \\
{[2.77 \%]}\end{array}$ \\
\hline & 1.00 & $2.10 \times 10^{-2}$ & $\begin{array}{c}1.96 \times 10^{-2} \\
{[-6.80 \%]}\end{array}$ & $\begin{array}{c}2.20 \times 10^{-2} \\
{[4.63 \%]}\end{array}$ \\
\hline & 2.00 & $1.09 \times 10^{-2}$ & $\begin{array}{c}9.90 \times 10^{-3} \\
{[-9.41 \%]}\end{array}$ & $\begin{array}{c}1.13 \times 10^{-2} \\
{[3.39 \%]}\end{array}$ \\
\hline \multirow{3}{*}{0.05} & 0.50 & $1.76 \times 10^{-1}$ & $\begin{array}{c}1.67 \times 10^{-1} \\
{[-5.10 \%]}\end{array}$ & $\begin{array}{c}1.80 \times 10^{-1} \\
{[2.65 \%]}\end{array}$ \\
\hline & 1.00 & $9.77 \times 10^{-2}$ & $\begin{array}{c}9.09 \times 10^{-2} \\
{[-6.88 \%]}\end{array}$ & $\begin{array}{c}1.02 \times 10^{-1} \\
{[4.51 \%]}\end{array}$ \\
\hline & 2.00 & $5.26 \times 10^{-2}$ & $\begin{array}{c}4.77 \times 10^{-2} \\
{[-9.48 \%]}\end{array}$ & $\begin{array}{c}5.44 \times 10^{-2} \\
{[3.27 \%]}\end{array}$ \\
\hline \multirow{3}{*}{0.1} & 0.50 & $3.02 \times 10^{-1}$ & $\begin{array}{c}2.86 \times 10^{-1} \\
{[-5.22 \%]}\end{array}$ & $\begin{array}{r}3.09 \times 10^{-1} \\
{[2.48 \%]}\end{array}$ \\
\hline & 1.00 & $1.79 \times 10^{-1}$ & $\begin{array}{c}1.67 \times 10^{-1} \\
{[-7.00 \%]}\end{array}$ & $\begin{array}{c}1.87 \times 10^{-1} \\
{[4.29 \%]}\end{array}$ \\
\hline & 2.00 & $1.01 \times 10^{-1}$ & $\begin{array}{c}9.12 \times 10^{-2} \\
{[-9.55 \%]}\end{array}$ & $\begin{array}{c}1.04 \times 10^{-1} \\
{[3.07 \%]}\end{array}$ \\
\hline
\end{tabular}

We investigate this reason in detail with an example case of $t_{p} / t=0.5, h / l=1$ used in Fig. 3 whose error becomes maximum. The error shown in Fig. 3 is

$$
\frac{E_{y, \text { dis }}-\left.E_{y, F E M}\right|_{\text {honeycomb }}}{\left.E_{y, F E M}\right|_{\text {honeycomb }}}=6.24 \%,
$$

where subscript $\left.\right|_{\text {honeycomb }}$ means the FEM analysis result of honeycomb sandwich to distinguish it from the FEM result of sandwich structure in Fig.7. The equivalent elastic modulus of 
Table 4 Young's modulus $E_{y} / E_{s}$ for regular hexagonal honeycomb with $E_{p} / E_{s}=2$ and $t_{p}=t_{1}=t_{2}=t$

\begin{tabular}{|c|c|c|c|c|}
\hline$t / l$ & $h / l$ & $E_{y, F E M} / E_{s}$ & $E_{y, c o m} / E_{s}$ & $E_{y, \text { dis }} / E_{s}$ \\
\hline \multirow{3}{*}{0.01} & 0.50 & $7.94 \times 10^{-2}$ & $\begin{array}{c}7.69 \times 10^{-2} \\
{[-3.14 \%]}\end{array}$ & $\begin{array}{c}8.02 \times 10^{-2} \\
{[0.98 \%]}\end{array}$ \\
\hline & 1.00 & $4.12 \times 10^{-2}$ & $\begin{array}{c}3.92 \times 10^{-2} \\
{[-4.78 \%]}\end{array}$ & $\begin{array}{c}4.18 \times 10^{-2} \\
{[1.55 \%]}\end{array}$ \\
\hline & 2.00 & $2.13 \times 10^{-2}$ & $\begin{array}{c}1.98 \times 10^{-2} \\
{[-7.23 \%]}\end{array}$ & $\begin{array}{c}2.14 \times 10^{-2} \\
{[0.12 \%]}\end{array}$ \\
\hline \multirow{3}{*}{0.05} & 0.50 & $3.45 \times 10^{-1}$ & $\begin{array}{c}3.34 \times 10^{-1} \\
{[-3.24 \%]}\end{array}$ & $\begin{array}{c}3.48 \times 10^{-1} \\
{[0.82 \%]}\end{array}$ \\
\hline & 1.00 & $1.91 \times 10^{-1}$ & $\begin{array}{c}1.82 \times 10^{-1} \\
{[-4.85 \%]}\end{array}$ & $\begin{array}{c}1.94 \times 10^{-1} \\
{[1.37 \%]}\end{array}$ \\
\hline & 2.00 & $1.03 \times 10^{-1}$ & $\begin{array}{c}9.55 \times 10^{-2} \\
{[-7.23 \%]}\end{array}$ & $\begin{array}{c}1.03 \times 10^{-1} \\
{[-0.01 \%]}\end{array}$ \\
\hline \multirow{3}{*}{0.1} & 0.50 & $5.92 \times 10^{-1}$ & $\begin{array}{c}5.73 \times 10^{-1} \\
{[-3.26 \%]}\end{array}$ & $\begin{array}{c}5.96 \times 10^{-1} \\
{[0.62 \%]}\end{array}$ \\
\hline & 1.00 & $3.52 \times 10^{-1}$ & $\begin{array}{c}3.35 \times 10^{-1} \\
{[-4.80 \%]}\end{array}$ & $\begin{array}{c}3.56 \times 10^{-1} \\
{[1.11 \%]}\end{array}$ \\
\hline & 2.00 & $1.98 \times 10^{-1}$ & $\begin{array}{c}1.84 \times 10^{-1} \\
{[-6.98 \%]}\end{array}$ & $\begin{array}{r}1.97 \times 10^{-1} \\
{[-0.17 \%]}\end{array}$ \\
\hline
\end{tabular}

sandwich structure of Fig.7 $E_{y, F E M}$ is calculated by FEM by taking the elastic modulus of each material as $E_{1} / E_{3}=E_{y, i} / E_{p}, E_{2} / E_{3}=E_{y, v} / E_{p}$, in which $E_{y, i}, E_{y, v}$ and $E_{p}$ are the equivalent elastic modulus of the inclined cell wall, the vertical cell wall and the face sheet respectively for the honeycomb. The error of Eq.(24) to $\left.E_{y, F E M}\right|_{\text {sandwich of Fig.(7) }}$ is

$$
\frac{E_{y, d i s}-\left.E_{y, F E M}\right|_{\text {sandwich of Fig.(7) }}}{\left.E_{y, F E M}\right|_{\text {sandwich of Fig.(7) }}}=2.51 \% \text {. }
$$

So, $2.51 \%$ is an error coming from the presented method of Eq.(24), and the rest comes from an antiplane warpage of face sheet and so on. The warpage can be seen from Fig.11. Figure 11 shows antiplane displacement $u_{z}$ of face sheet from $\mathrm{B}$ to $\mathrm{C}$ for the example model used in Fig.2. Though the antiplane displacement is very small, existence of it can be confirmed from the figure. So, an error will yield in the strain energy of face sheet obtained from Eq.(23) which doesn't consider the antiplane warpage. In addition, a similar deformation can also be seen in the cell wall of core. The deformation of inclined cell wall needs a bending rotation around a direction other than $z$-direction due to the core deformation, which is given by Eq.(21), and is inhomogeneous along the height, and then an error occurs because of new strain energy accompanying with this deformation.

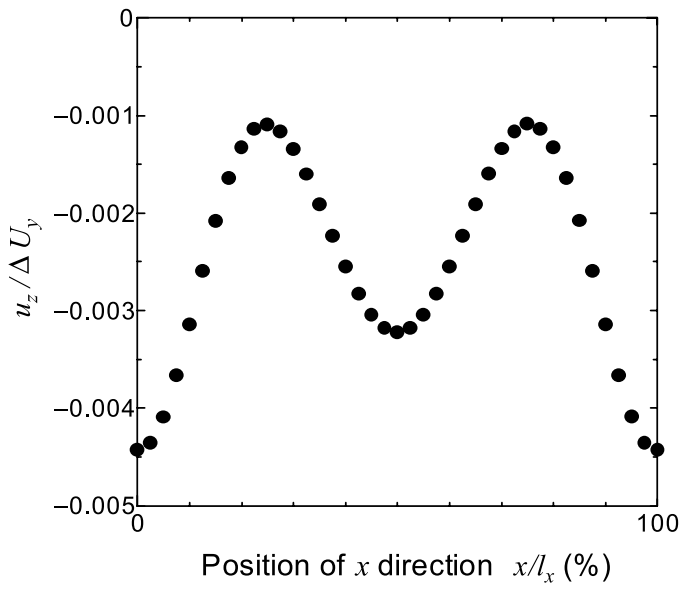

Fig. 11 Antiplane displacement of face sheet 


\section{Conclusions}

In this paper, an analysis of in-plane equivalent elastic modulus $E_{y}$ of honeycomb sandwich was studied by considering interference effect of the core with the face sheet and the following conclusions are obtained.

(1) Errors in assessing the equivalent elastic modulus $E_{y, c o m}$ of a honeycomb sandwich by the law of mixture can become more than $20 \%$ depending on material and geometry size. The reason of the error is because the rigidity to deformation of $y$-direction is different greatly between the vertical cell wall of core and inclined cell wall of core.

(2) A new analysis method of equivalent elastic modulus $E_{y}$ is presented, in which the interference effect between the core and face sheet is taken into account by considering the honeycomb as a mixture consisting of three parts with different elastic properties: part of inclined cell wall, part of vertical cell wall and part of face sheet. The validity of presented method for determining the equivalent elastic modulus $E_{y}$ is verified by comparing their results with numerical results of FEM.

( 3 ) The accuracy of the presented method is better than that of the law of mixture. However, the analysis accuracy may become lower. Especially, the error becomes more than 5\%, when the face sheet is so thin that $t_{p} / t \leq 0.5$. The reason why the accuracy deteriorates is because of the antiplane warpage of face sheet. So, the warpage of face sheet must be considered to improve the analysis accuracy of equivalent elastic modulus $E_{y}$ for a honeycomb sandwich with thin face sheet.

\section{References}

( 1 ) I.G. Masters and K.E. Evans, Models for the elastic deformation of honeycombs, Compos. Struct., 35, (1996), 403-422.

( 2 ) W. E. Warren and A. M. Kraynik, The linear elastic response of two-dimensional spatially periodic cellular materials, Mechanics of Materials, 6(1), (1987), 27-37.

( 3 ) L. J. Gibson and M. F. Ashby, Cellular Solids: Structures \& Properties, Pergamon Press, Oxford, (1988), 89-93.

( 4 ) D.H. Chen, H. Horii and S. Ozaki, Analysis of In-plane Elastic Modulus for a Hexagonal Honeycomb Core (1st, Effect of Core Height and Proposal of Analytical Method), JSME, A48, (2007), 18-25.

( 5 ) W. Becker, The in-plane stiffnesses of a honeycomb core including the thickness effect, Archives of Applied Mechanics, 68, (1998), 334-341.

( 6 ) W. Becker, Closed-form analysis of the thickness effect of regular honeycomb core material, Compos. Struct., 48, (2000), 67-70.

( 7 ) J. Hohe and W. Becker, A refined analysis of the effective elasticity tensor for general cellular sandwich cores, Int. J. Solids Struct., 38, (2001), 3689-3717.

( 8 ) A. Chen and J. F. Davalos, A solution including skin effect for stiffness and stress field of sandwich honeycomb core, Int. J. Solids Struct., 42, (2005), 2711-2739.

( 9 ) D.Hull, Composite Materials, Cambridge University Press, England, (1982), Chapter 5.

(10) MSC. Marc, User's Manual, (2003). 